\title{
Comparative evaluation of rK9, rK26 and rK39 antigens in the serodiagnosis of Indian visceral leishmaniasis
}

\author{
Tribhuban Mohan Mohapatra ${ }^{1}$, Dharmendra Prasad Singh ${ }^{1}$, Malay Ranjan Sen ${ }^{1}$, Kalpana \\ Bharti ${ }^{1}$ and Shyam Sundar ${ }^{2}$ \\ ${ }^{1}$ Department of Microbiology and ${ }^{2}$ Department of Medicine, Institute of Medical Sciences, BHU, VARANASI-221005, India
}

\begin{abstract}
Background: This study was designed for comparative evaluation of two relatively newer recombinant hydrophilic antigens, rK9 and rK26 of Leishmania chagasi along with rK39 (a 39-aminoacid-repetitive immunodominant B-cell epitope of kinesin-related antigen from L. chagasi) and crude soluble antigen (CSA) for the serodiagnosis of Indian visceral leishmaniasis (VL) patients by quantitative ELISA.

Methodology: In the present study a total of 80 subjects comprising of 55 confirmed VL cases and 25 endemic controls (EC) were subjected to ELISA using four different antigens, namely rK9, rK26, rK39 and CSA (derived from Leishmania donovani promastigotes).

Results: Sensitivity was as follows: $78 \%$ (95\%CI 63-100\%) for rK9, 38\% (95\%CI 28-59\%) for rK26, 100\% for rK39, and 80\% (95\% CI 65$100 \%$ ) for CSA. The specificity of rK9, rK26, rK39 and CSA was found to be 84\% (95\%CI 61-100\%), 80\% (95\%CI 56-100\%), 96\% (95\%CI $75-100 \%)$ and $72 \%(95 \% \mathrm{CI} 49-100 \%)$, respectively.

Conclusions: rK39 was observed to be the most suitable antigen as compared to rK26 and rK9 whereas rK9 performed better than rK26. Hence rK9 antigen may either be used as an adjunct to rK39 for accurate diagnosis of VL or may be used in the absence or non-availability of rK39 antigen for the serodiagnosis.
\end{abstract}

Key words: rK9, rK26, rK39, visceral leishmaniasis, crude soluble antigen, serodiagnosis

J Infect Dev Ctries 2010; 4(2):114-117.

(Received 30 September 2009 - Accepted 19 October 2009)

Copyright $\odot 2010$ Mohapatra et al. This is an open-access article distributed under the Creative Commons Attribution License, which permits unrestricted use, distribution, and reproduction in any medium, provided the original work is properly cited.

\section{Introduction}

Visceral leishmaniasis (VL) is a potentially fatal disease caused by Leishmania donovani complex comprised of $L$. donovani (Indian subcontinent and Africa), L. chagasi (South America) and L. infantum (Mediterranean countries). The disease is endemic in 88 countries on 5 continents with a total of 350 million people at risk and12 million cases [1]. More than $90 \%$ of cases are from India, Nepal, Bangladesh, southern Sudan and northeast Brazil.

Detection of the parasite is definitive as well as the gold standard for the diagnosis of VL. But the procedure is cumbersome, risky and very difficult to apply in field conditions. Conventional methods of antibody detection have become obsolete as the sensitivities and specificities of most of the tests have been the limiting factors [2-4]. In addition, various serological tests such as IFAT, DAT, KATEX and ELISA have been developed, evaluated and tried [510]. ELISA is the best choice for the development of a rapid and reliable diagnostic method, because it is more practical, easy to standardize and suitable for mass screening. Specificity and sensitivity of the ELISA based immunoassay strictly depends on antigen quality and can be improved by use of recombinant technology [6], which drives the expression and purification of diagnostically relevant proteins in large amounts [11].In the last decade, several Leishmania antigens have been genetically and antigenically characterized. Recombinant K39 antigen (rK39) is a 39-amino-acid repetitive immunodominant B-cell epitope of the $230 \mathrm{kDa}$ kinesin related protein of $L$. chagasi $[12,13]$. The rK39 ELISA has been demonstrated to be suitable for detection of human VL and canine (both clinical and asymptomatic) VL [12-14].

Recently, reported cloning and characterisation of two new related hydrophilic antigens of Leishmania chagasi, rK9 and rK26, have led to an increase in the list of recombinant antigens for serodiagnosis of VL. K9 and K26 differ in the presence of 11 copies of a 14-amino-acid repeat in the open reading frame of K26 [15]. 
The aim of the present study was to assess the performance of rK9 and rK26 antigens for the serodiagnosis of VL and to compare the results with rK39 and CSA by quantitative ELISA.

\section{Materials and methods}

Sera

Sera from 55 clinically diagnosed as well as parasitologically confirmed VL cases were collected from Sir Sunder Lal Hospital, Banaras Hindu University, and KAMRC, its field site at Muzaffarpur, Bihar. Informed consent was obtained prior to blood collection. None of the VL patients had received any treatment before the collection of blood. The sera were separated and preserved at $-70^{\circ} \mathrm{C}$ until use.

\section{Selection Criteria}

Power analysis was utilized for calculation of sample size. Only those clinically suspected patients whose diagnosis of VL was confirmed by demonstration of LD bodies in splenic aspirates were included in the study. Endemic controls (EC) were selected as healthy volunteers or relatives of $\mathrm{VL}$ patients (no history of VL/relapse of VL) living in the area of endemicity (Muzaffarpur). All the subjects were screened for HIV infection by tri-dot test and found to be negative.

\section{ELISA}

The recombinant antigens (rK9, rK26 and rK39) were provided by Dr. S. G. Reed of Corixa Corporation, Seattle, WA, U.S.A. The tests were performed following the method described by Kumar et al. [16]. Briefly, 96-well microtitre plates were coated with rK9 (25 ng) or rK26 (25 ng) or rK39 (25 ng) or $5 \mu \mathrm{g}$ of CSA per well. Protein (crude soluble fraction) of promastigotes [MHOM/IN/01/BHU36] isolated from an Indian patient with VL was used as CSA. After coating, plates were kept overnight at $4^{\circ} \mathrm{C}$ then aspirated, blocked with PBS containing 1 or $5 \%$ (wt/vol) bovine serum albumin (BSA) for 2 hours at room temperature and then washed six times with PBS containing $0.1 \%$ Tween 20 (PBS-T). Sera were serially diluted in PBS containing 0.1\% BSA (1\% when tested for CSA). Next $0.1 \%$ Tween 20 was added to the wells, and plates were incubated for 30 minutes at room temperature for rK9, rK26 and rK39 and for one hour at $37^{\circ} \mathrm{C}$ for CSA. The wells were then washed six times with PBS-T and incubated for 30 minutes with protein A-horseradish peroxidase (1/2000 dilution; Bangalore Genei, India) in PBS containing $0.1 \%$ BSA and $1.1 \%$ Tween 20 for one hour at $37^{\circ} \mathrm{C}$ for rK9, rK26 and rK39. The conjugates antisera for CSA was goat anti-human immunoglobulin $G$ conjugated with horseradish peroxidase $(1 / 5,000$ dilution). Plates were then washed six times in PBS-T and incubated with tetramethylbenzidine (TMB) substrate for a further 15 minutes for recombinant antigens and $30 \mathrm{~min}$ for CSA. The reaction was stopped by the addition of $1 \mathrm{~N}$ sulphuric acid, and the optical density at $450 \mathrm{~nm}$ was determined. The cutoff points for rK9, rK26, rK39 and CSA were determined from the serum dilution with optical densities of $0.102,0.106,0.101$ and 0.346 , respectively, calculated as the mean of the negative controls plus 3 standard deviations. All the sera were tested on single dilution (1:512) and OD was estimated. Sera having cutoff values more than that of the respective antigen as described earlier were further tested for quantitative ELISA. To quantitatively assess the result for each serum, the highest dilution giving the positive reaction were read by the naked eye and expressed as titers. Each serum was assayed in triplicate with negative (healthy individual from non-endemic area) and positive serum samples used as standards.

\section{Results}

Sera from 43 out of 55 patients $(78 \%)$ showed high titers $\left(2.5 \times 10^{3}-5.2 \times 10^{5}\right)$ with rK9. Only 21 out of 55 patients $(38 \%)$ showed high titer $\left(1.2 \times 10^{3}-1.6 \times\right.$ $\left.10^{4}\right)$ with rK26. All patients $(100 \%)$ showed very high titers $\left(6.5 \times 10^{4}-1.0 \times 10^{6}\right)$ with rK39 and 44 out of $55(80 \%)$ patients showed high titers $\left(2.7 \times 10^{3}\right.$ to $4.0 \times 10^{4}$ ) with CSA. The range of antibody titers from the controls was $1.0 \times 10^{3}-1.5 \times 10^{4}$ for $\mathrm{rK} 9$, $0.8 \times 10^{3}-5.1 \times 10^{3}$ for rK26, $3.5 \times 10^{3}-1.7 \times, 10^{4}$ for $\mathrm{rK} 39$ and $0.9 \times 10^{3}-4.0 \times 10^{3}$ for CSA. The mean titer for detecting antibodies to different antigens is shown in table 2. Statistical evaluation was done after entry into the EPI-info (version-6) and SPSS statistical packages for calculation of sensitivity and specificity. Sensitivity was found to be $78 \%$ (95\% CI $63-100 \%$ ) for rK9, 38\% (95\% CI $28-59 \%)$ for $\mathrm{rK} 26,100 \%$ for rK39, and $80 \%$ (95\% CI $65-100 \%)$ for CSA. Specificity was found to be $84 \%$ (95\%CI 61-100\%) for rK9, 80\% (95\% CI 56-100\%) for rK26, 96\% (95\% CI 75-100\%) for rK39 and 72\% (95\% CI 49-100\%) for CSA and, as shown in table 1.

\section{Discussion}

Classic symptomatic VL is almost always fatal if left untreated. Definitive diagnosis of VL still 
Table 1. Sensitivity and Specificity of Antigens.

\begin{tabular}{|l|c|c|}
\hline Antigens & Sensitivity & Specificity \\
\hline CSA & $80 \%(95 \%$ CI 65-100\%) & $72 \%(95 \%$ CI 49-100\%) \\
\hline rK9 & $78 \%(95 \%$ CI 63-100\%) & $84 \%(95 \%$ CI 61-100\%) \\
\hline rK 26 & $38 \%(95 \%$ CI 28-59\%) & $80 \%(95 \%$ CI 56-100\%) \\
\hline rK 39 & $100 \%$ & $96 \%(95 \%$ CI 75-100\%) \\
\hline
\end{tabular}

Table 2. Mean Titer of CSA, rK9, rK26, and rK39 for detection of antibody by ELISA.

\begin{tabular}{|l|l|l|l|l|l|}
\hline Subjects & Total & \multicolumn{4}{|c|}{ Mean Titer } \\
\cline { 3 - 6 } & no. & CSA & rK9 & rK26 & rK39 \\
\hline VL & 55 & $1.8 \times 10^{3}$ & $6.2 \times 10^{3}$ & $1.4 \times 10^{3}$ & $3.1 \times 10^{5}$ \\
\hline EC & 25 & $1.1 \times 10^{3}$ & $1.4 \times 10^{3}$ & $1.0 \times 10^{3}$ & $6.5 \times 10^{3}$ \\
\hline
\end{tabular}

depends on parasite detection in a smear or culture of aspirate from the spleen, bone marrow or lymph node. These tests can be insensitive or generate equivocal results [17]. In addition, the procedure must be performed by individuals with the required expertise or the tests may result in serious consequences [17]. Noninvasive tests, such as conventional detection of $\operatorname{IgG}$ antibodies of Leishmania, are often not sensitive enough to detect asymptomatic or subclincial individuals [12]. Though serological tests such as IFAT, ELISA using CSA, and CIEP have become available during last few years, due to various reasons none of them have proved to be as reliable as the splenic smear [18]. Consequently, there is a need for a specific as well as sensitive serological test/s to diagnose the disease.

A total of $55 \mathrm{VL}$ cases and $25 \mathrm{EC}$ were investigated for antibody response to rK9, rK26, rK39 and CSA to determine the most suitable antigen for diagnostic purposes. The sensitivity and specificity of rK9 and rK26 detected in this preliminary study are supported by another study conducted in India which reported $21.3 \%$ sensitivity by the rK26 strip test [19]. The difference in sensitivity might be that a quantitative method was used in the current study whereas the rK26 strip test used in the other study is a qualitative method of antibody detection. Other studies conducted in symptomatic canine VL cases in Brazil showed higher sensitivity by ELISA using rK9 and rK26 antigen [20-22]. The lower sensitivity in this study may be due to the fact that dog is a different species than human. Another explanation may be that there are different leishmanial species (other than $L$. donovani) causing canine Leishmaniasis; hence difference in the antibody response. In this study, rK39 antigen showed a sensitivity of $100 \%$ and specificity of $96 \%$. Similar findings have also been observed by other researchers [23]. Another investigation showed great utility of rK39 in unambiguous diagnosis and prognosis of VL which was much superior to crude soluble antigen [14]. On analysis, though the specificities of both rK9 and rK26 were comparable ( $84 \%$ \& $80 \%$, respectively), the sensitivity of $\mathrm{rK} 9$ was much higher $(78 \%)$ than rK26 (38\%).

This study was conducted on limited cases; hence no definite conclusion can be drawn. From the preliminary study, it appears that for the serological diagnosis, rK39 is the most suitable antigen followed by rK9 and rK26. Pending detailed serological evaluation of rK9 with large samples and wellmatched controls, we believe that this antigen could be used as an adjunct to parasitological diagnosis or in the absence/unavailability of rK39 for an effective, non-invasive and accurate diagnosis of VL in remote endemic areas.

\section{Acknowledgements}

The authors would like to thank Dr. S. G. Reed of Corixa Corporation, Seattle, USA, for providing rK9, rK26 and rK39 antigens for serological evaluation. This work was supported by a WHO-RCS grant.

\section{References}

1. WHO (1991) Leismania/HIV co-infection. Weekly Epidemiological Record 72: 49-56.

2. Bray RS (1976) Immunodiagnosis of leishmaniasis. In S Cohen and EH Sadun editors. Immunology of Parasitic Infections. Oxford: Blackwell Scientific Publications, 65-76.

3. Hockmeyer WT, Wellde BT, Sabwa CL, Smith DH, Rees PH, Kager PA (1984) A complement fixation test for visceral leishmaniasis using homologous parasite antigen. Ann Trop Med Parasitol 78: 489-493.

4. Aikat BK, Sehgal S, Mahajan RC, Pathania AG, Bhattacharya PK, Sahaya S, Choudhury AB, Pasricha N, Prasad LS (1979) The role of counter-electrophoresis as a diagnostic tool in kala-azar. Indian J Res Med 70: 592-597. 
5. Duxbury RE and Sadun EH (1964) Fluorescent Antibody Test for the serodiagnosis of visceral leishmaniasis. Am J Trop Med Hyg 13: 525-529.

6. Hommel M (1976) Enzyme immunoassay in leishmaniasis. Trans R Soc Trop Med Hyg 70: 15-16.

7. Attar ZJ, Chance ML, el-Safi S, Carney J, Azazy A, El Hadi M, Dourado C, Hommel M (2001) Latex agglutination test for the detection of urinary antigens in visceral leishmaniasis. Acta Trop 78: 11-16.

8. Meredith SE, Kroon NC, Sondorp E, Seaman J, Goris MG, Van Ingen CW, Oosting $\mathrm{H}$, Schoone GJ, Terpstra WJ, Oksam L (1995) Leish-KIT, a stable direct agglutination test based on free dried antigen for the serodiagnosis of visceral leishmaniasis. J Clin Microbiol 33: 1742-1745.

9. Sundar S, Kumar K, Singh VP, Mohapatra TM (1991) Prolonged diagnostic lag period in Kala-azar test for early diagnosis needed. JAPI 39: 651.

10. Zijlstra EE, Osman OF, Hofland HWC, Oskam L, Ghalib HW, El-Hassan AM, Kager PA, Meredith SEO (1997) The direct agglutination test for diagnosis of visceral leishmaniasis under field conditions in Sudan: comparison of aqueous and freeze dried antigens. Trans R Soc Trop Med Hyg 91: 671-673.

11. Sundar S, Rai M (2002) Laboratory diagnosis of visceral leishmaniasis. Clin Diagn Lab Immunol 9: 951-958.

12. Badaro R, Benson D, Eulalio MC, Freire M, Cunha S, Netto EM, Pedral-SampaioD, Madureira C, Burns JM, Houghton RL, David JR, Reed SG (1996) rK39: a cloned antigen of Leishmania chagasi that predicts active visceral leishmaniasis. J Infect Dis 173: 758-761.

13. Burns JM Jr, Shreffler WG, Benson DR, Ghalib HW, Badaro R, Reed SG (1993) Molecular characterization of a kinesin-related antigen of Leishmania chagasi that detects specific antibody in African and American visceral leishmaniasis. Proc Natl Acad Sci USA 90: 775-779.

14 Qu J-Q, Zhong L, Masoom-Yasinzai M, Abdur-Rab M, Aksu, HSZ, Reed SG, Chang KP, Gilman-sachs A (1994) Serodiagnosis of Asian leishmaniasis with a recombinant antigen from the repetitive domain of a Leishmania kinesin. Trans Soc Trop Med Hyg 88: 543-545.

15. Bhatia A, Daifalla NS, Jen S, Badaro R, Reed SG, Skeiky YA (1999) Cloning,characterization and serological evaluation of K9 and K26: two related hydrophilic antigens of Leishmania chagasi. Mol Biochem Parasitol 102: 249261.

16. Kumar R, Pai K, Pathak K, Sundar S (2001) Enzyme-linked immunosorbent assayfor rK39 antigen in diagnosis and prognosis of Indian visceral leishmaniasis. Clin Diagn Lab Immunol 8: 1220-1224.

17. Siddig M, Ghalib H, Shillington DC, Peterson EA (1988) Visceral leishmaniasis in Sudan: a comparative parasitological method of diagnosis. Trans Roy Soc Trop Med Hyg 82: 66-68.

18. Sundar S, Singh GS, Mohapatra TM, Singh VP, Raj VS, Vinayak VK, Singla N (1994) Immunodiagnosis of kalaazar with special reference to DAT. Tropical diseases molecular biology and control strategies: In Kumar S, Sen AK, Dutta GP, Sharma RN, editors. New Delhi: Publication and Information Directorate, CSIR. 459-462.

19. Sundar S, Singh RK, Bimal SK, Gidwani K, Mishra A, Maurya R, Singh SK, Manandhar KD, Boelaert M, Rai M (2007) Comparative evaluation of parasitological and serological tests in the diagnosis of visceral leishmaniasis in India: a phase III diagnostic accuracy study. Trop Med Int Health 12: 284-289.

20. Renato Porrozzi, Marcos V Santos da Costa, Antonio Teva, Aloisio Falqueto, Adelson L Ferreira, Claudiney D dos Santos, Ana Paula Fernandes, Ricardo T Gazzinelli, Antonio Campos-Neto, Gabriel Grimaldi Jr. (2007) Comparative evaluation of ELISA based on crude and recombinant leishmanial antigen for serodiagnosis of symptomatic and asymptomatic Leishmanial infantum visceral infections in dogs. Clin and Vaccine immunol14: 544-548.

21. Roberto teodoro da costa, Joao Carlos Franca, Wilson Mayrink, Evaldo Nascimento, Odair Genaro, Antonio campos-Neto (2003) Standardization of a rapid immunochromatographic test with the recombinant antigens K39 and K26 for the diagnosis of canine visceral leishmaniasis. Tropical Medicine and Hygiene 97: 678-682.

22. Rosati S, Ortoffi M, Profiti M, Mannelli A, Mignone W, Bollo E, Gradoni L (2003) Prokaryotic expression and antigenic characterization of three recombinant Leishmania antigens for serological diagnosis of canine leishmaniasis. Clin Dign Lab Immunol 10: 1153-1156.

23. Sundar S, Reed SG, Singh VP, Kumar PCK, Murray HW (1998) Rapid accurate field diagnosis of Indian visceral leishmaniasis. Lancet 351: 563-565.

\section{Corresponding Author}

Prof. T. M. Mohapatra

Tel: \#91 542 2369328, \#91 9450530467

Fax: \#91 5422367568

E mail:tmmohapatra2000@yahoo.com

Conflict of interest: No conflict of interest is declared. 